\title{
Fingerprint Enhancement Based on Discrete Cosine Transform
}

\author{
Suksan Jirachaweng and Vutipong Areekul \\ Kasetsart Signal \& Image Processing Laboratory (KSIP Lab), \\ Department of Electrical Engineering, Kasetsart University, Bangkok, 10900, Thailand \\ \{g4885038, fengvpa\}@ku.ac.th \\ http://ksip.ee.ku.ac.th
}

\begin{abstract}
This paper proposes a novel fingerprint enhancement algorithm based on contextual filtering in DCT domain. All intrinsic fingerprint features including ridge orientation and frequency are estimated simultaneously from DCT analysis, resulting in fast and efficient implementation. In addition, the proposed approach takes advantage of frequency-domain enhancement resulting in best performance in high curvature area. Comparing with DFT domain, DCT has better signal energy compaction and perform faster transform with real coefficients. Moreover, the experimental results show that the DCT approach is out-performed the traditional Gabor filtering, including the fastest separable Gabor filter, in both quality and computational complexity.
\end{abstract}

Keywords: Fingerprint Enhancement, Discrete Cosine Transform Enhancement, Frequency-Domain Fingerprint Enhancement.

\section{Introduction}

Inevitably, many fingerprint identification applications are playing an important role in our everyday life from personal access control, office time attendance, to country boarder control. To pursue this goal, automatic fingerprint identification system (AFIS) must be proved to be highly reliable. Since most automatic fingerprint identification systems are based on the minutiae and ridge matching, these systems rely on good quality of input fingerprint images for minutiae and ridge extraction. Unfortunately, bad quality of fingerprint and elastic distortion are now major problems for most AFISs especially large database systems. In order to reduce the error accumulated from false accept rate and false reject rate, quality of fingerprint must be evaluated and enhanced for better recognition results.

Based on filtering domains, most fingerprint enhancement schemes can be roughly classified into two major approaches; i.e. spatial-domain and frequency-domain. Filtering in spatial-domain applies convolution directly to fingerprint image. On the other hand, filtering in frequency-domain need Fourier analysis and synthesis. Fingerprint image is transformed, then multiplied by filter coefficients, and inverse transformed Fourier coefficients back to enhanced fingerprint image. In fact if employed filters are the same, enhancement results from both domains must be exactly the same by signal processing theorem. However, for practical implementation, these 
two approaches are different in terms of enhancement quality and computational complexity of algorithms.

Practical performing fingerprint enhancement based on each domain has different advantage and disadvantage. For example, most popular Hong's Gabor filters [1], with orientation and frequency spatially adaptable, are applied to partitioning fingerprint image. However, this Gabor filter model is based on unidirectional ridge enhancement, resulting in ridge discontinuity and blocking artifacts around highly curvature region. On the other hand, for frequency domain approaches, natural fingerprint image is localized in some frequency coefficients. Gabor filter can be easily designed to cooperate with high curvature area. For example, Kamei et al. [2] introduced fingerprint filter design based on frequency domain using discrete Fourier transform. Chikkerur et al. [3] applied short time Fourier transform and took advantage from 2-dimensional filter shaping design, adapted with highly curvature area, resulting in better enhanced results. However, comparing with spatial-domain approaches, this scheme suffers from high computational complexity in Fourier analysis and synthesis even though Fast Fourier Transform (FFT) is employed.

In order to take advantage from frequency-domain fingerprint enhancement with low computational complexity, we propose fingerprint enhancement based on Discrete Cosine Transform (DCT). The DCT is a unitary orthogonal transform with real coefficients. It is closely related to the Discrete Fourier transform (DFT) which has complex coefficients. Moreover, it has been known that DCT provides a distinct advantage over the DFT in term of energy compaction and truncation error [4]. Thus is why DCT has been widely employed in general image and video compression standards. Hence, in this paper, we investigated DCT-base fingerprint enhancement for practical implementation. We expected best enhanced quality results with low computational complexity. This paper is organized as follows. Section 2 describes several processes in order to implement enhancement filtering in DCT domain including intrinsic estimation and practical filtering. Section 3 shows experimental evaluation. Finally, section 4 concludes our works and future research.

\section{Proposed Approach}

The fingerprint enhancement approach consists of 4 concatenated processes; i.e. discrete cosine transform of sub-blocks of partitioning fingerprint, ridge orientation and frequency parameters estimation, filtering in DCT domain, and inverse discrete cosine transform of sub-blocks. The advantages of the proposed approach are as follows.

- Fingerprint ridges form a natural sinusoid image, which its spectrums are packed or localized in frequency domain. Hence these spectrums can be easily shaped or filtered in this domain. Moreover, filter can be specially designed in order to handle high curvature ridge area such as singular points. This is the great advantage over the spatial-domain filtering approach.

- Comparing with discrete Fourier transform, discrete cosine transform performs better in term of energy compaction. Moreover, DCT coefficients are real number comparing with complex number of DFT coefficients. Therefore, we can handle DCT coefficients easier than DFT coefficients. Besides, fast DCT 
requires less computational complexity and less memory usage comparing with fast Fourier transform (FFT).

- By partitioning fingerprint into sub-blocks, the proposed approach utilizes spatially contextual information including instantaneous frequency and orientation. Intrinsic features such as ridge frequency, ridge orientation, and angular bandwidth can be simply analyzed directly from DCT coefficients.

Each process of the proposed fingerprint enhancement is explained as follows.

\subsection{Overlapping DCT Decomposition and Reconstruction}

Conventional fingerprint enhancement schemes, applying with non-overlapping blocks of partitioning fingerprint, often encounter with blocking artifacts such as ridge discontinuity and spurious minutiae. To preserve ridge continuity and eliminate blocking artifacts, overlapping block is applied to both DCT decomposition and reconstruction, similar to the DFT approach in [3]. However, there is no need to apply any smooth spectral window for DCT because overlapping area is large enough to prevent any blocking effects, corresponding with its energy compaction property.

\subsection{Intrinsic Parameter Estimation on DCT Domain}

Ridge frequency, ridge orientation, and angular bandwidth can be analyzed from DCT coefficients directly. Therefore DCT analysis yields appropriate domain to perform fingerprint enhancement and provides filtering parameters as the same time.

Ridge Frequency Estimation: The ridge frequency $\left(\rho_{0}\right)$ is simply obtained by measuring a distance between the origin $(0,0)$ and the highest DCT peak of highfrequency spectrum as following equation,

$$
\rho_{0}=\sqrt{u_{0}^{2}+v_{0}^{2}}
$$

where $\left(u_{0}, v_{0}\right)$ is the coordinate of the highest peak of high-frequency spectrum.

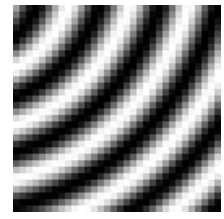

(a)

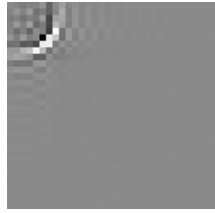

(b)

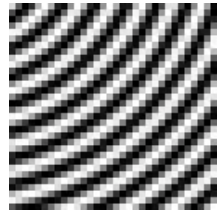

(c)

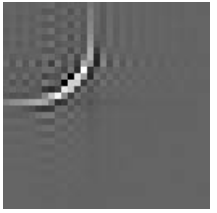

(d)

Fig. 1. Figure (a) and (c) represent blocks of a fingerprint model with different frequency. Figure (b) and (d) are DCT coefficients of figure (a) and (c), respectively. Note that DC coefficient is set to zero in order to clearly display high-frequency spectrum.

Ridge orientation estimation: The dominant orientation of parallel ridges, $\theta$, are closely related to a peak-angle, $\phi$, in DCT coefficients, where $\phi$ is measured counterclockwise (if $\phi>0$ ) from the horizontal axis to the terminal side of the highest spectrum peak of high frequency (DC spectrum is not included). However, $\theta$ and $\phi$ relationship is not one-to-one mapping. The ridge orientation, which $\theta$ varies in the 
range of 0 to $\pi$, is projected into the peak-angle, which $\phi$ varies in the range of 0 to $\pi / 2$. Relationship between $\theta_{0}$ ridge orientation in spatial domain and $\phi_{0}$ peak angle in frequency domain are described in equation (2) with some examples in Fig. 2.

$$
\phi_{0}=\tan ^{-1}\left(\frac{v_{0}}{u_{0}}\right), \quad \phi_{0}=\left|\frac{\pi}{2}-\theta_{0}\right| \text { where } 0 \leq \theta_{0} \leq \pi
$$

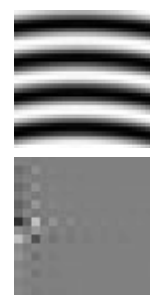

$\theta_{0}=\pi=0$

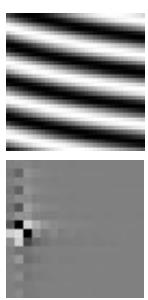

$\theta_{0}=7 \pi / 8$

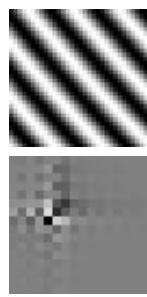

$\theta_{0}=3 \pi / 4$

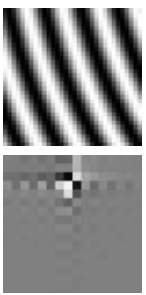

$\theta_{0}=5 \pi / 8$

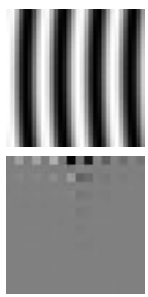

$\theta_{0}=\pi / 2$

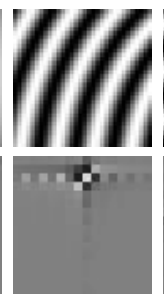

$\theta_{0}=3 \pi / 8$

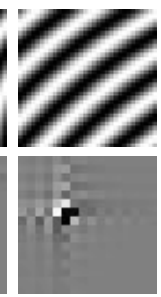

$\theta_{0}=\pi / 4$

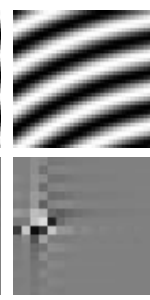

$\theta_{0}=\pi / 8$

Fig. 2. Examples of relationship between ridge orientation in spatial domain and peak-angle in DCT domain, all ridge angles refer to horizontal axis and DC coefficient is set to zero in order to show high-frequency spectrum. (Note that only the top-left quarters of DC coefficients are zoomed in for clear view of high-frequency peak behavior.)

From Fig. 2, ridge orientation at $\pi-\theta$ has the highest spectrum peak with the same location as ridge orientation at $\theta$. However, their phase patterns are distinguishable by observation. Therefore additional phase analysis is needed to classify the quadratics of ridge orientation in order to correctly perform fingerprint enhancement. Since Lee et al. [5] proposed edge detection algorithm based on DCT coefficients, our fingerprint enhancement modified Lee's approach by modulation theorem in order to detect quadrant of fingerprint ridge orientation.

According to Lee's technique, the orientation quadrant of a single line can be determined by the polarities of two first AC coefficients, $G_{01}$ and $G_{10}$, where $G_{u v}$ is the

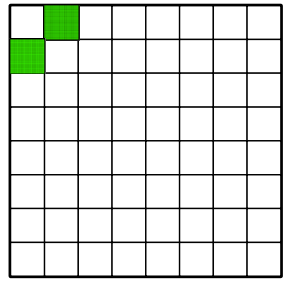

(a)

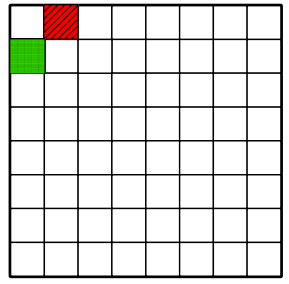

(b)

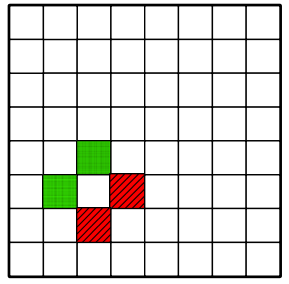

(c)

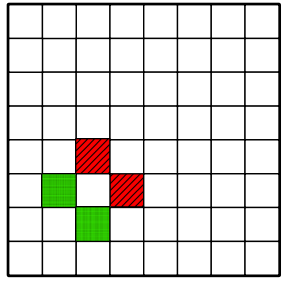

(d)

Fig. 3. Four polarity patterns indicate (a) a single line orientation ranging from 0 to $\pi / 2$, (b) a single line orientation ranging from $\pi / 2$ to $\pi$, (c) parallel ridge orientation ranging from 0 to $\pi / 2$, and (d) parallel ridge orientation ranging from $\pi / 2$ to $\pi$ 
DCT coefficient at coordinate $(u, v)$, as shown in Fig. 3. In case of a single line, polarity of product of $G_{01}$ and $G_{10}$ coefficients indicates the line orientation. If $\mathrm{G}_{01} \times \mathrm{G}_{10} \geq 0$, this line orientation is in the first quadrant (0 to $\left.\pi / 2\right)$ as shown in Fig. 3(a). On the other hand, if $\mathrm{G}_{01} \times \mathrm{G}_{10}<0$, this line orientation is in the second quadrant $(\pi / 2$ to $\pi)$ as shown in Fig. 3(b). This technique can be applied to detect orientation of parallel lines or ridges by modulation theorem with the pattern of polarities around the high peak DCT coefficients. To be precise, ridge orientation in the first quadrant ( 0 to $\pi / 2$ ) and ridge orientation in the second quadrant ( $\pi / 2$ to $\pi$ ) can be indicated by the same polarities of $45^{\circ}$ and $135^{\circ}$ diagonal coefficients referred to the highest absolute peak as shown in Fig. 3(c) and (d), respectively.

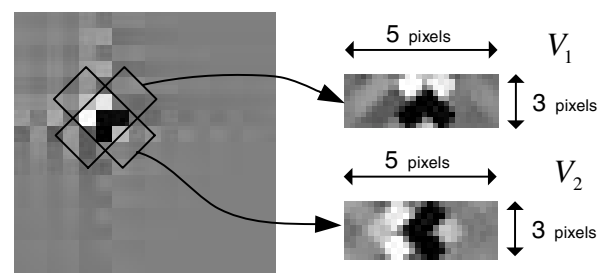

Fig. 4. Demonstrate 2-D perpendicular diagonal vectors, $V_{1}$ at $45^{\circ}$ and $V_{2}$ at $135^{\circ}$, referred to the highest absolute spectrum peak (the center black pixel (negative value))

In order to identify the quadrant and avoid influence of interference, two 2-D perpendicular diagonal vectors, $V_{1}$ and $V_{2}$, are formed with size of $5 \times 3$ pixels, center at the peak position as shown in Fig. 4. The average directional strengths of each vector $\left(S_{1}, S_{2}\right)$ are then computed by equation (3). Then the quadrant can be classified and the actual fingerprint ridge orientation can be identified as shown in equation (4).

$$
\begin{aligned}
S_{i}=\underset{n=-1,0,1}{\operatorname{Max}} \frac{\left|\sum_{m=-2}^{2} V_{i}\left(u_{0}+m, v_{0}+n\right)\right|}{5} \text { where } i=1,2 \\
\theta= \begin{cases}\pi / 2-\phi & \text { where } S_{1} \geq S_{2} \\
\pi-(\pi / 2-\phi) & \text { Otherwise }\end{cases}
\end{aligned}
$$

Finally, the estimated ridge frequency and orientation of each local region is formed a frequency field and an orientation field. Then Gaussian filter is applied to smooth both global fields in order to reduce noise effect as [1].

Angular bandwidth estimation: At the singularity region, ridge spectrum is not an impulse but it spreads bandwidth out. Therefore, the desired filter of each block must be adapted based on its angular bandwidth. We slightly modified the coherence parameter from Chikkerur's concept in [3], called non-coherence factor. This noncoherence factor represents how wide ridge orientation can be in the block that has more than one dominant orientation. This factor is in the range of 0 to 1 , where 1 represents highly non-coherence or highly curved region and 0 represents uniorientation region. The non-coherence factor can be given by 


$$
N C\left(u_{c}, v_{c}\right)=\frac{\sum_{(i, j) \in W}\left|\sin \left(\theta\left(u_{c}, v_{c}\right)-\theta\left(u_{i}, v_{j}\right)\right)\right|}{W \times W}
$$

where $\left(u_{c}, v_{c}\right)$ is the center position of block, $\left(u_{i}, v_{j}\right)$ is the $i^{\text {th }}$ and $j^{\text {th }}$ positions of neighborhood blocks within $W \times W$, and the angular bandwidth, $\phi_{B W}$, can be estimated by the equation (6) as follows,

$$
\phi_{B W}\left(u_{c}, v_{c}\right)=\sin ^{-1}\left(N C\left(u_{c}, v_{c}\right)\right) .
$$

\subsection{Enhancement Filtering in DCT Domain}

In DCT domain, filtering process is not simply as in DFT domain [2,3], which required only coefficient multiplication. The Gabor filter in [1] is modified in order to cooperate with DCT domain based on Cartesian-form representation. The enhancement filtering in DCT domain can be separated into two arithmetic manipulation; i.e. multiplication and convolution.

1) Filtering by Multiplication: The enhancement filter can be expressed in term of product of separable Gaussian functions, similar to the frequency-domain filtering technique in [2] as follows.

$$
F_{f d}(\rho, \phi)=F(\rho, \phi) H_{f}(\rho) H_{d}(\phi)
$$

where $F(\rho, \phi)$ is DCT coefficients in polar-form representation, directly related to DCT coefficients, $F(u, v)$, in rectangular-form representation. $F_{f d}(\rho, \phi)$ is DCT coefficients of the filtering output. The $H_{f}(\rho)$ filter, which performs the ridge frequency filtering.in Gaussian shape, is given by

$$
H_{f}\left(\rho \mid \rho_{0}, \sigma_{\rho}, Z\right)=\frac{1}{Z} \exp \left(-\frac{\left(\rho-\rho_{0}\right)^{2}}{2 \sigma_{\rho}^{2}}\right), \rho_{0}=\sqrt{u_{0}^{2}+v_{0}^{2}} ; \rho_{\min } \leq \rho_{0} \leq \rho_{\max }
$$

where $\rho_{0}$ and $\sigma_{\rho}$ are the center of the high-peak frequency group and the filtering bandwidth parameter, respectively. The $\rho_{\min }$ and $\rho_{\max }$ parameters are minimum and maximum cut-off frequency constraints, which suppress the effects of lower and higher frequencies such as ink, sweat gland holes, and scratches in the fingerprint. The $Z$ is a filtering normalization factor, depending on filtering energy result.

The $H_{d}(\phi)$ filter, which performs the ridge orientation filtering, is given by

$$
H_{d}\left(\phi \mid \phi_{0}, \sigma_{\phi}, \phi_{B W}\right)=\left\{\begin{array}{cl}
\exp \left(-\frac{\left(\phi-\phi_{0}\right)^{2}}{2 \sigma_{\phi}^{2}}\right) & \text { where }\left|\phi-\phi_{0}\right| \geq \phi_{B W} \\
1 & \text { Otherwise }
\end{array}\right.
$$

where the $\phi_{0}$ is the peak orientation for bandpass filter, $\sigma_{\phi}$ is the directional bandwidth parameter, and $\phi_{B W}$, the angular bandwidth, is given by equation (6). 
2) Filtering by Convolution: Since the $\theta$ and $\pi-\theta$ ridge orientation coefficients are projected into the same DCT-domain region. Therefore, both directional coefficients still remain from the previous filtering. In order to truncate inappropriate directional coefficients, two diagonal Gabor filters are exploited by convolution operation. The finally enhanced DCT coefficients are given by

$$
F_{E n h}(u, v)=F_{f d}(u, v) * H_{q}(u, v)
$$

where $F_{E n h}(u, v)$ is enhanced DCT coefficients in rectangular-form. $F_{f d}(u, v)$ is the previous result of enhanced DCT coefficients in rectangular-form, by converted from $F_{f d}(\rho, \phi)$ in polar-form. The quadrant correction filter, $H_{q}(u, v)$, is given by

$$
H_{q}(u, v)=\left\{\begin{array}{l}
\cos \left[\frac{(u+v) \pi}{2}\right] \exp \left(-\frac{(u+v)^{2}}{2 \sigma_{q}^{2}}\right) \text { where } \theta \geq \pi / 2 \\
\cos \left[\frac{(u-v) \pi}{2}\right] \exp \left(-\frac{(u-v)^{2}}{2 \sigma_{q}^{2}}\right) \text { Otherwise }
\end{array}\right.
$$

where $\sigma_{q}$ is the quadratic parameter and $\cos (n \pi / 2)$ only has three values $-1,0$ and -1 . Indeed, this convolution operation requires low computation because most of bandpass filtered coefficients are truncated to zero from the previous operation. In case of highly curved ridges, the transformed coefficients are projected into widely curved subband of DCT domain as shown in Fig. 5.
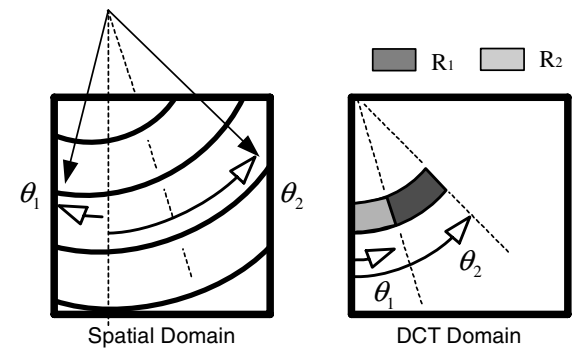

Fig. 5 Highly curved ridges in spatial and frequency (DCT) domain. Signal is localized in widely curved subband, which can be classified into the principal region $\left(R_{1}\right)$ and the reflection region $\left(R_{2}\right)$.

From Fig. 5, we approximate the orientation range from $\theta_{1}$ to $\theta_{2}$ by non-coherence factor from the equation (6). The curved subband can be classified into two regions; i.e. principal region $\left(R_{1}\right)$ and reflection region $\left(R_{2}\right)$. The principal region $\left(R_{l}\right)$ contains only one diagonal component $\left(45^{\circ}\right.$ or $\left.135^{\circ}\right)$ as mentioned before. The $45^{\circ}$ or $135^{\circ}$ diagonal components are the phase pattern of the oriented ridges in the range of $0^{\circ}$ to $90^{\circ}$ or $90^{\circ}$ to $180^{\circ}$, respectively. The reflection region $\left(R_{2}\right)$ composes of both of $45^{\circ}$ and $135^{\circ}$ diagonal components from the reflection property of DCT coefficients. Then the convolution is applied only in the principal region. 


\section{Experimental Evaluation}

The experimental results have been evaluated on public fingerprint database FVC2002 Db3a [6] (100 users, 8 images each) in term of enhancement quality, matching performance, and computational complexity. The fingerprint image is partitioned into blocks of $16 \times 16$ pixels, and a simple segmentation scheme using mean and variance is employed. Five fingerprint enhancement filtering types are evaluated as follows; Traditional Gabor filtering with non-quantized orientation (TG)[1], Separable Gabor filtering with non-quantized orientation (SG)[7], Separable Gabor filtering with 8-quantized orientation (SG8)[8], Short-Time Fourier Transform approach (STFT)[3], and proposed approach (DCT). In the spatial domain approaches, the discrete Gabor filters are the same $25 \times 25$ fixed-window size. Note that the separable Gabor filter [7,8] was implemented on the fly using a set of priori created and stored filters. Moreover, symmetric of 2-D Gabor filter [1] was also exploited in this process. These filtering schemes accelerated execution speed of the traditional Gabor enhancement process as fast as possible. For the STFT [3] and the DCT approaches in frequency domain, fingerprint image is also partitioned into $16 \times 16$ blocks but each block is transformed with $32 \times 32$ overlapped window to reduce blocking artifacts. Note that the probability estimation in [3] is not included.

In order to compare the performance of various enhancement algorithms, three evaluation methodologies are used; i.e. the goodness index [1] of minutiae extraction, the matching performance, and the average execution time. First, the goodness index $(G I)$ from [1] is employed to measure the extracted minutiae quantity from each fingerprint enhancement algorithm. In this case, we needed to manually mark minutiae of all fingerprints in FVC2002 Db3a. The goodness index is given by

$$
G I=\frac{\sum_{i=1}^{r} q_{i}\left[M_{i}-L_{i}-S_{i}\right]}{\sum_{i=1}^{r} q_{i} T_{i}},
$$

where $r$ is the number of $16 \times 16$ windows in the input fingerprint image, $q_{i}$ represents the quality factor of $i^{\text {th }}$ window $(\operatorname{good}=4$, medium $=2$, poor $=1$ ) which estimated by partitioning and thresholding of the dryness factor (mean $\times$ variance of block) and the smudginess factor (mean / variance of block). $M_{i}$ represents the number of minutiae pair, which match with human expert in a tolerance box in the $i^{\text {th }}$ window. $L_{i}$ and $S_{i}$ represent the number of lost and spurious minutiae in the $i^{\text {th }}$ window, respectively. $T_{i}$ represents the number of minutiae extracted by experts.

Second, enhancement results are tested with our minutiae matching verification algorithm based on Jiang's concept of [9], and the equal error rate (EER) is reported. Finally, the average execution time of fingerprint enhancement process is measured for FVC2002 Db3a (image size $300 \times 300$ pixels) on Pentium M $1.5 \mathrm{GHz}$ with $376 \mathrm{Mb}$ RAM. Note that execution time includes filter parameter estimation (frequency and orientation), transform (if required), and filtering process. However, segmentation process is not included and we used the same segmentation process for all comparison schemes. The objective test results are summarized in Table 1. Contradict to our belief; overall execution time of DCT approach is faster than the separable Gabor 
Table 1. Summary of the performance comparison among various fingerprint enhancement algorithms over FVC 2002 Db3a Fingerprint Database, Pentium M 1.5GHz, 376Mb RAM

\begin{tabular}{lccc}
\hline $\begin{array}{l}\text { Fingerprint Enhancement } \\
\text { Algorithm }\end{array}$ & $\begin{array}{c}\text { Average } \\
\text { Goodness } \\
\text { Index (GI) [1] }\end{array}$ & $\begin{array}{c}\text { Our } \\
\text { Matching } \\
\text { (\% EER) }\end{array}$ & $\begin{array}{c}\text { Execution } \\
\text { Time } \\
\text { (Second) }\end{array}$ \\
\hline TG [1] & 0.160 & 9.716 & 0.973 \\
SG [7] & 0.167 & 9.326 & 0.278 \\
SG8 [8] & 0.181 & 12.196 & 0.160 \\
STFT (modified from [3]) & 0.250 & 7.713 & 0.172 \\
DCT (Proposed Approach) & 0.336 & 6.846 & 0.151 \\
\hline
\end{tabular}

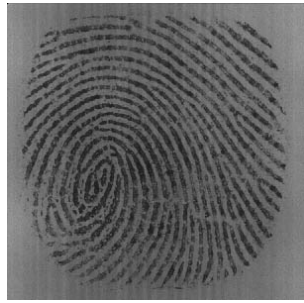

$\left(\mathbf{a}_{1}\right) \# 20 \_5$

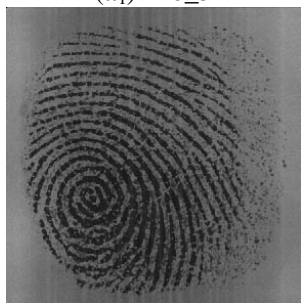

$\left(\mathbf{a}_{2}\right) \# 40 \_4$

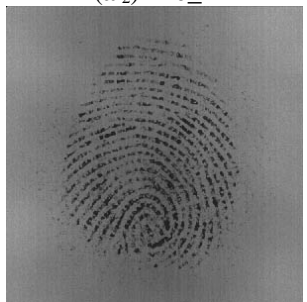

$\left(\mathbf{a}_{3}\right) \# 107 \quad 7$

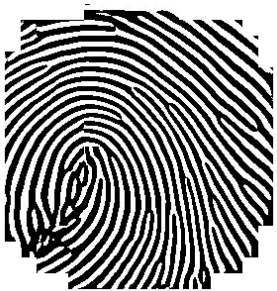

$\left(\mathbf{b}_{1}\right) \mathrm{SG}[7](\mathrm{GI}=0.59)$

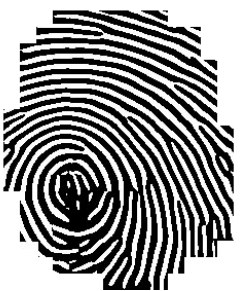

$\left(\mathbf{b}_{2}\right) \mathrm{SG}[7](\mathrm{GI}=0.19)$

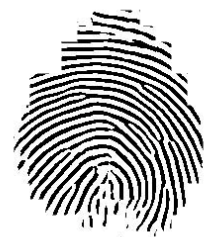

(b $\left.{ }_{3}\right)$ SG[7] $(\mathrm{GI}=0.18)$

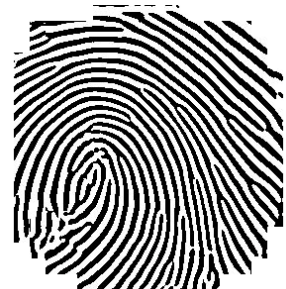

$\left(\mathbf{c}_{1}\right)$ STFT[3] (GI=0.63)

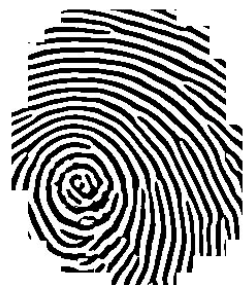

$\left(\mathbf{c}_{2}\right) \operatorname{STFT}[3](\mathrm{GI}=0.30)$

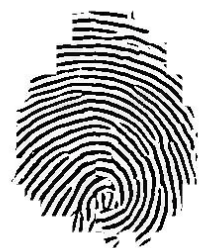

(c) $\operatorname{STFT}[3](\mathrm{GI}=0.47)$

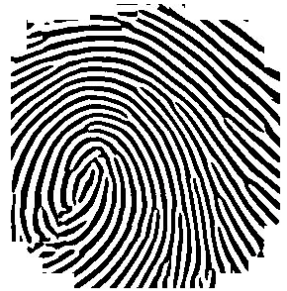

$\left(\mathbf{d}_{1}\right) \operatorname{DCT}(\mathrm{GI}=0.70)$

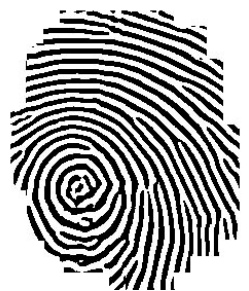

$\left(\mathbf{d}_{2}\right) \operatorname{DCT}(\mathrm{GI}=0.32)$

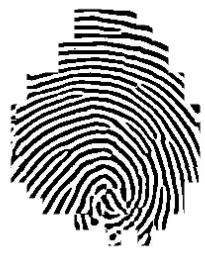

$\left(\mathbf{d}_{3}\right) \operatorname{DCT}(\mathrm{GI}=0.68)$

Fig. 6. (a) Original fingerprint \#20_5, \#40_4 and \#107_7 from FVC2002 Db3a, (b) Enhanced results from SG[7], (c) Enhanced results from STFT modified from [3], (d) Enhanced results of our proposed DCT based method

filtering with 8-quantized orientation. We investigated in depth and we found that even though separable 2-D convolution alone is faster than both FFT and Fast DCT analysis and synthesis, the fingerprint intrinsic parameter estimation was slow this approach down since these parameters are evaluated in frequency domain.

Fig. 6 shows enhancement results for subjective tests with GI values for objecttive tests. Note that the quality of enhanced fingerprints is improved based on 
frequency-domain filtering, especially in highly curved ridges. Overall of FVC2002, DB3a database, both STFT and DCT based performed very well around highly curved area with slightly different results around singular point area.

\section{Conclusion and Future Research}

In conclusion, this paper proposes a novel fingerprint enhancement approach based on discrete cosine transform (DCT). The enhancement takes advantage of filtering real DCT coefficients with high-energy compaction in frequency-domain. Hence filtering can be specially designed to cooperate highly curvature area resulting in less discontinuity and blocking artifacts comparing with spatial-domain filtering.

For future research, we will conduct exhaustive experiments based on all FVC databases in order to prove the efficient of DCT-based fingerprint enhancement. To achieve this goal, all minutiae in all FVC databases need to be manually marked. We will also exploit orientation adaptive filter in DCT Domain in the near future.

Acknowledgments. This work was partially supported by Department of Electrical Engineering, Kasetsart University, Thailand Research Fund (TRF) through the Royal Golden Jubilee Ph.D. Program (Grant No.PHD/0017/2549), and the Commission on Higher Education through the TRF Research Scholar (Grant No. RMU4980027).

\section{References}

1. Hong, L., Wang, Y., Jain, A.K.: Fingerprint Image Enhancement: Algorithm and Performance Evaluation. IEEE Trans. on Pattern Analysis and Machine Intelligence 20(8), 777-789 (1998)

2. Kamei, T., Mizoguchi, M.: Image Filter Design for Fingerprint Enhancement. In: Proc. ISCV'95, pp. 109-114 (1995)

3. Chikkerur, S., Cartwright, A.N., Govindaraju, V.: Fingerprint Enhancement Using STFT Analysis. Pattern Recognition 40, 198-211 (2007)

4. Rao, K.R., Yip, P.: Discrete Cosine Transform: Algorithms, Advantages, Applications. Academic Press, Boston, MA (1990)

5. Lee, M., Nepal, S., Srinivasan, U.: Role of edge detection in video semantics. In: Proc. PanSydney Workshop on Visual Information Processing (VIP2002). Conferences in Research and Practice in Information Technology, Australia (2003)

6. Maltoni, D., Maio, D., Jain, A.K., Prabhakar, S.: Fingerprint Verification Competition 2002. Database Available: Handbook of Fingerprint Recognition. Springer, Heidelberg (2003)

7. Areekul, V., Watchareeruetai, U., Suppasriwasuseth, K., Tantaratana, S.: Separable Gabor filter realization for fast fingerprint enhancement. In: Proc. Int. Conf. on Image Processing (ICIP 2005), Genova, Italy, pp. III-253-III-256 (2005)

8. Areekul, V., Watchareeruetai, U., Tantaratana, S.: Fast Separable Gabor Filter for Fingerprint Enhancement. In: Zhang, D., Jain, A.K. (eds.) ICBA 2004. LNCS, vol. 3072, pp. 403-409. Springer, Heidelberg (2004)

9. Jiang, X., Yau, W.Y.: Fingerprint Minutiae Matching Based on the Local and Global Structures. In: Proc. Int. Conf. on Pattern Recognition (15 $\left.{ }^{\text {th }}\right)$, vol. 2, pp. 1042-1045 (2000) 